\title{
1-508-4
}

\section{Epigenetic mechanisms of stress resilience and susceptibility and its implications for drug development}

\section{Shusaku Uchida}

Kyoto Univ, MIC

Although stressful events predispose individuals to psychiatric disorders, such as depression, not all people who encounter a stressful life experience become depressed, suggesting that gene-environment interactions (GxE) determine depression risk. Accumulating evidence has implicated that stress-induced aberrant synaptic and structural plasticity may be key underlying mechanisms of stress susceptibility. Recent evidence has provided key insights into the biological significance of epigenetic regulation of gene expression in synaptic plasticity and behavioral response to chronic stress. The medial prefrontal cortex $(\mathrm{mPFC})$ is vulnerable to damage from a variety of psychosocial stressors and aberrant structural and functional changes in this brain structure have been implicated in depression. However, little is known about the role of epigenetic mechanisms within the $\mathrm{mPFC}$ in chronic stress-induced aberrant neuronal plasticity and depression-like behavior. In this symposium, I will focus on causal and mechanistic evidence implicating altered functions and connectivity of the mPFC circuits in the establishment and the maintenance of stress resilience and susceptibility. I also touch upon recent findings suggesting a role for epigenetic mechanisms in these processes and briefly discuss promising avenues of future investigation. 